\title{
Parainfluenza Virus Type-3 Outbreak in Level II Neonatal Care Unit: Role of Nursing Infants inside Closed Incubators in the Control of the Viral Outbreak
}

\author{
Khalil Al Tawil $^{1, *}$, Saif Alsaif ${ }^{1}$, Atef Alshafei ${ }^{1}$, Vivian Manzano ${ }^{1}$, Ali Hajeer ${ }^{2}$, Hesham Tawakol ${ }^{1}$ \\ ${ }^{1}$ Department of Pediatrics, King Saud Bin Abdulaziz University for Health Sciences, King Abdulaziz Medical City, Riyadh, KSA \\ ${ }^{2}$ Department of Molecular Biology Lab, King Saud Bin Abdulaziz University for Health Sciences, King Abdulaziz Medical City, \\ Riyadh, KSA \\ *Corresponding author: tawilk@ngha.med.sa
}

Received October 08, 2014; Revised October 29, 2014; Accepted November 04, 2014

\begin{abstract}
Parainfluenza type 3 virus (PIV-3) outbreaks in neonatal care units are rare. We aimed to report our experience of PIV-3 outbreak in level II neonatal care unit (NCU-II). A retrospective review of medical records of all infants managed in NCU-11 during the PIV-3 outbreak period. During the viral outbreak, 49 infants were cared for in NCU-II, and only 7 infants proved to be infected with PIV-3. The attack rate was $14 \%$. The first 4 infected infants were transferred to standby isolation room outside NCU-II, and the unit was closed. All exposed and infected infants were cohorted, nursed inside closed incubators and other infection control measures were reinforced. Due to bed crises and 5 days after the closure of the unit and before the end of the viral outbreak; the standby isolation room was closed and NCU-II was reopened for new admissions and was divided into 3 zones for: infected, exposed, and newly admitted infants. Three more infants that were initially exposed to index case turned positive. There was no further transmission of the PIV-3 after opening the neonatal unit. Infants nursed in open crib at onset of PIV-3 outbreak were at high risk for infection (P value $<0.0001$ ). All infected infants survived and were discharged in good condition. Re-enforcing standard infection control measures, cohorting and placing all the exposed and infected infants inside closed incubators could contain respiratory outbreaks in neonatal nurseries without significant morbidity or mortality and most probably without the need to close the unit.
\end{abstract}

Keywords: parainfuenza virus, neonatal care unit, incubators, open cribs, infection control

Cite This Article: Khalil Al Tawil, Saif Alsaif, Atef Alshafei, Vivian Manzano, Ali Hajeer, and Hesham Tawakol, "Parainfluenza Virus Type-3 Outbreak in Level II Neonatal Care Unit: Role of Nursing Infants inside Closed Incubators in the Control of the Viral Outbreak.” Journal Name, vol. 2, no. 5 (2014): 117-121. doi: 10.12691/ajidm-2-5-4.

\section{Introduction}

Parainfluenza virus (PIV) is an enveloped RNA paramyxovirus with four distinct subtypes. It causes more than $30 \%$ of all acute respiratory infections in infants and children [1]. It is only second to respiratory syncytial virus as a cause of lower respiratory tract infection in young children [2-3]. Of the four serotypes of human PIV, type-3 causes the majority of infections in this age group. Although highly infective, outbreaks of PIV-3 in nursery accounts for $1-3 \%$ of all cases of viral infections in neonatal care unit [4]. PIV-3 outbreaks in infants are rare probably due to presence of maternal antibodies which are transferred antenatally through placenta [5] and possibly postnatally through breast milk [6]. Premature infants might be more susceptible because of the insufficiency of transferred maternal antibodies secondary to the premature delivery.

Some of the previously reported PIV-3 outbreaks in newborn nurseries were difficult to control without closing the unit while others were associated with mortalities [6,7]. We are describing an outbreak of PIV-3 in NCU-II which was successfully contained with use of infection control measures and nursing exposed and infected infants inside closed incubators without prolonged closure of the unit and with no significant morbidity or mortality.

\section{Material and Methods}

A retrospective review of the medical records of all infants who were managed in the NCU-II during the PIV3 outbreak period from April 26 to May 7, 2008 at King Abdul-Aziz Medical City-Riyadh, a tertiary care center in Riyadh, Saudi Arabia. The NCU-II is a rectangular, 28 bedded unit that receives 1200 admission per year and is separate from level III neonatal intensive care unit.

We studied the demographic data, clinical presentation, management, and outcome of all the involved infants during the viral outbreak. The demographic data of the infected and uninfected infants were compared to study the possible risk factors for the PIV-3 outbreak. 


\subsection{Statistics}

Statistical analysis was done using STATA program version 11.1 (2009), STATA corporation LP, TX, USA. Student-t test was used for analysis of continuous data and Chi Square Test was used for analysis of categorical data. $P$ value less than 0.05 was considered to be significant.

\subsection{Patients}

Patients were considered symptomatic if they developed any of the following: coryza, cough, recurrent apneas, retraction, wheezes, bradycardia, tachypnia or desaturation. Symptomatic infants were placed in closed incubators and given intravenous antibiotics for 48 hours pending the bacterial culture result. Infants with apnea or unstable oxygen saturation were given oxygen by nasal cannula, and were initiated on intravenous fluids. Salbutamol nebulization was administrated only to wheezing infants. Patients who needed mechanical ventilation support were transferred to the fully equipped Standby Isolation Room outside NCU-II.

Infants on the right and left side of any symptomatic baby were considered exposed and they were nursed inside closed incubators. Unless symptomatic all other infants were considered unexposed. Infected infants were considered recovered if they tested negative on the PIV-3 antigen detection.

\subsection{Laboratory Studies}

Nasopharyngeal swabs were obtained from symptomatic and exposed infants and were sent to the laboratory on the Universal Viral Transport Media. The swabs were tested for PIV-3 antigen detection by direct immune fluorescence antibody using the Ultra PARA3 DFA reagent with or without viral culture using the RMix SV/CS. All the used laboratory products were obtained from Diagnostic Hybrids Inc, CA, USA. Positive nasopharyngeal swabs were repeated every third day until turned negative for the infected infants while the exposed infants were rescreened twice during the outbreak duration. Chest X-ray and other investigations were done when clinically indicated.

\subsection{Control Measures}

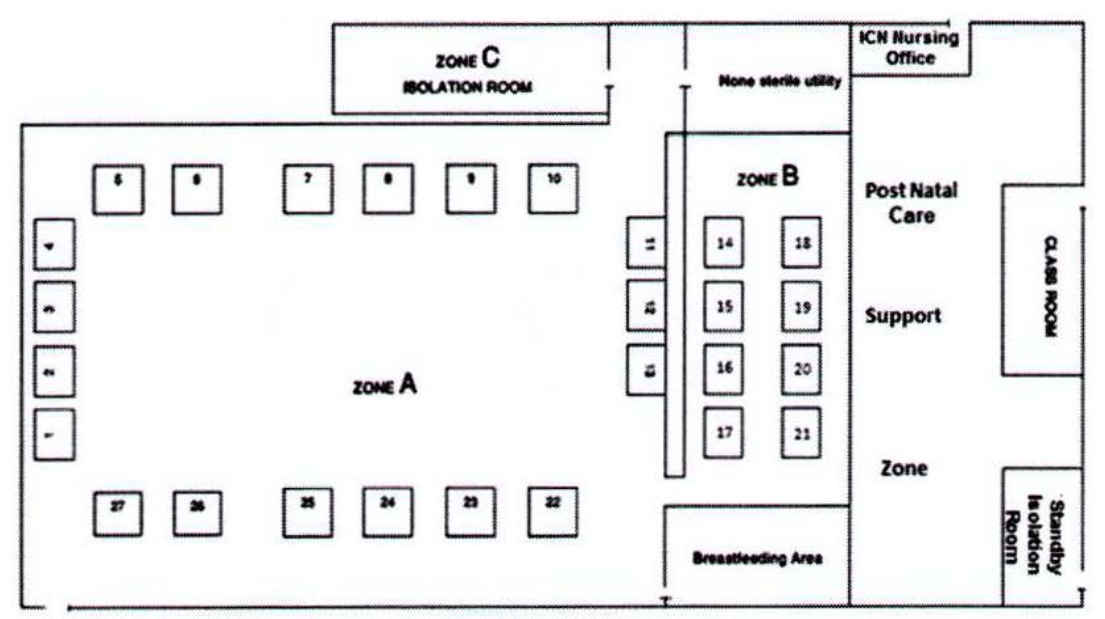

Figure 1. Structure and distribution of patients in the Neonatal Care Nursery-II during the Parainfluenza-3 Virus Octbreak 


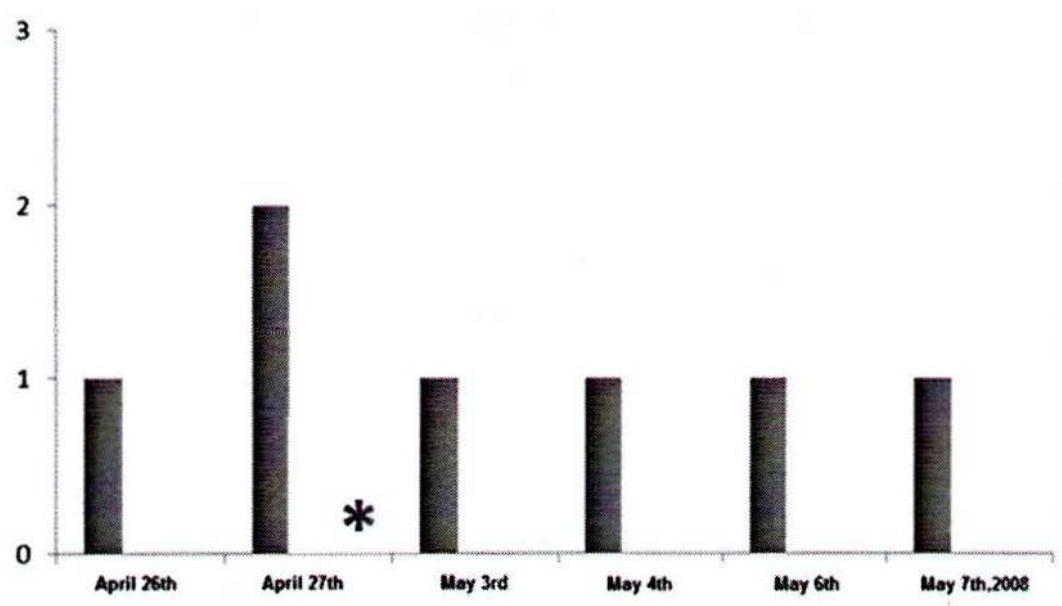

The Unit was closed for new admissions between April 28th and May 3rd, 2008

Figure 2. Distribution of parainfluenza-3 virus cases between April 26th-May 7th 2008

All the infected infants were in the group of infants who were exposed to the index case of outbreak, and at the onset of outbreak were nursed in open incubators. All newly admitted babies during the two periods of opening the unit stayed asymptomatic.

The duration of symptoms ranged from 1 to 6 days with a median of 3 days, the duration of positivity ranged from 2 to 14 days with a median of 7 days. All symptomatic infants in the infected group were positive for PIV- 3 by antigen detection as well as cultures. Apnea developed in 5 infected infants but only in one infant in the noninfected group. Only one infected infant needed nasal intermittent mandatory ventilation for 5 days and was managed in the standby isolation room outside NCU-II. All the other infants were treated with only bronchodilators and other supportive care, antiviral medications were not prescribed for any infant. All the infants survived and were discharged in a good condition. The clinical symptoms of the infected infants are shown in Table 2.
Table 1. Demographic characteristics of all infants admitted to NCU-11 at the time of PIV-3 viral outbreak

\begin{tabular}{|l|l|l|l|}
\hline & $\begin{array}{l}\text { Non- Infected } \\
(\mathrm{n}=42)\end{array}$ & $\begin{array}{l}\text { Infected } \\
(\mathrm{n}=7)\end{array}$ & P Value \\
\hline Birth weight (Kg) & & & \\
Mean & 2.61 & 1.67 & 0.0093 \\
Median & 2.5 & 1.4 & \\
\pm SD* & 0.81 & 0.82 & \\
\hline Gestational age(weeks) & & & \\
Mean & 36.86 & 31 & 0.0032 \\
Median & 37 & 30 & \\
\pm SD & 3.38 & 4.43 & \\
\hline Postnatal age (days) & & & \\
Mean & 5.64 & 60.1 & $<0.0001$ \\
Median & 1.0 & 37.0 & \\
\pm SD & 10.45 & 44.57 & \\
\hline NCU-II days & & & \\
Mean & 2.8 & 26 & $<0.0001$ \\
Median & 1.0 & 14 & \\
\pm SD & 3.3 & 36.4 & \\
\hline
\end{tabular}

*SD: Standard Deviation

Table 2. Clinical Manifestation of PIV-3 Infected Compared to Non-infected Infants

\begin{tabular}{|l|l|l|l|l|}
\hline Nasal secretion & $100 \%(7)$ & $2.4 \%(1)$ & $287(12.1-13175.5)$ & $<0.0001$ \\
\hline Cough & $57.1 \%(4)$ & $00 \%(00)$ & $54.7(3.3-2750.4)$ & $<0.0001$ \\
\hline Wheezes & $42.9 \%(3)$ & $00 \%(00)$ & $30.8(1.7-1650.2)$ & 0.0003 \\
\hline Tachypnea & $71.4 \%(5)$ & $00 \%(00)$ & $102.5(5.8-4947.1)$ & $<0.0001$ \\
\hline Change in oxygen requirement & $42.9 \%(3)$ & $2.4 \%(1)$ & $15(1.9-118.1)$ & 0.0021 \\
\hline
\end{tabular}

No viral screening was done for the nursing staff, 3 history of invasive mechanical ventilation, nursed in open nurses were referred to employee health clinic because of respiratory tract symptoms. They were granted a sick leave with a strict recommendation not to come back to work unless asymptomatic. No sick parents were identified during that period.

The characteristics of the 2 groups were studied regarding: history of non- invasive mechanical ventilation, crib prior to outbreak, broncho-pulmonary dysplasia, history of systemic steroid therapy, chronic non respiratory problems for the identification of possible risk factors and all proved to reach statistical significance as possible risk factors with significant $\mathrm{P}$ value of less than 0.05 as shown in Table 3 .

Table 3. Possible Risk Factors of PIV-3 Infected Compared to Non-infected Infants

\begin{tabular}{|l|l|l|l|l|}
\hline & $\begin{array}{l}\text { Infected } \\
\%(\mathrm{n}=7)\end{array}$ & $\begin{array}{l}\text { Non-Infected } \\
\%(\mathrm{n}=42)\end{array}$ & OR (95\% CI) & P Value \\
\hline History of non- invasive mechanical ventilation & $71.4 \%(5)$ & $23.8 \%(10)$ & $8(1.1-91.4)$ & 0.01 \\
\hline History of invasive mechanical ventilation & $71.4 \%(5)$ & $2.4 \%(1)$ & $102.5(5.8-4947)$ & $<0.0001$ \\
\hline Nursed in open crib prior to outbreak & $100 \%(7)$ & $14.3 \%(6)$ & $42(3.8-1941.8)$ & $<0.0001$ \\
\hline Broncho-pulmonary dysplasia & $28.6 \%(2)$ & $2.4 \%(1)$ & $16.4(0.7-989.8)$ & 0.0075 \\
\hline History of systemic steroid therapy & $28.6 \%(2)$ & $2.4 \%(1)$ & $16.4(0.7-989.8)$ & 0.0075 \\
\hline Chronic non respiratory problems ** & $42.9 \%(3)$ & $7.1 \%(3)$ & $9.8(0.9-94.6)$ & 0.0076 \\
\hline
\end{tabular}

** Osteopenia of Prematurity, Retinopathy of Prematurity, Obstructive Genito-Urinary Disorders, Hydrocephalus, Chronic seizures disorders. 


\section{Discussion}

Viral outbreaks in neonatal nurseries could cause serious morbidity and mortality. The most common reported respiratory viral outbreaks are due to respiratory syncytial virus (RSV). Overcrowding, prematurity, and noncompliance with infection control precautions play a major role in the spread of these viruses [8].

Although PIV-3 outbreaks are rare in neonatal nurseries partly because of the high reported frequency of passive immunity to PIV in the neonatal period from maternal antibodies [5].The attack rate in our report was 14\%, which is very low in comparison to other reports $[7,9]$. The increased susceptibility of NCU-II population could be due to the faded maternal neutralizing antibodies secondary to the long stay of premature infants in such nurseries [7]. The duration of the outbreak was 12 days; much shorter than other reports [10]. In concordance with Ben -Shimolet al report[11]; we encountered very low(14.3\%) morbidity as only one infant needed mechanical respiratory support in the form of Nasal IMV, compared to other reports of $71.4 \%$ of infected infants needed mechanical respiratory support [10] and had no mortality in comparison to significant mortality reported by others[6].

The incubation period of PIVs lasts from 2 to 4 days. The immunocompetent children may shed the virus for up to 1 week before onset of clinical infection and up to 3 weeks after the resolution of symptoms. Similar to the observation of Ben- Shimol et al, at the onset of the viral outbreak; all infected infants compared to $13.9 \%$ of the control ( $\mathrm{P}$ value $<0.0001$ ) were nursed in open crib. This finding suggests that infants nursed in open cribs are at high risk of infection at viral outbreak [11]. Transmission of parainfluenza infection occurs by direct person to person contact as well as inhalation of infected droplets $[12,13]$. Parainfluenza viruses have been found to survive for at least a few hours in air and on environmental surfaces [14,15]. Infectious viruses can be transferred from environmental surfaces to the hands of hospital personnel who appear to be responsible for the spread [13]. Nursing infectious infants inside closed incubators during parainfluenza viral outbreaks will prevent the transfer of the virus to surrounding environmental surfaces and thereby to the hands of health care providers, who may spread it to other patients. Such practice is the standards of practice in the control of RSV outbreaks in neonatal units [16]. During the viral outbreak in our NCU-II, we carried out a bundle of infection control measures (cohorting the infected and exposed infants in separate zones, placed all the infected and exposed infants inside closed incubators, grouping the nurses to care for infants in only one zone, and emphasizing hand washing and all the other infection control precautions) that enabled our team to contain the outbreak without prolonged closure of the unit. Despite the fact that our unit was partially closed i.e. the unit was closed for 5 days only, which is less than the period of expected infectivity of the PIV-3 virus, all the infected infants were in the group which was exposed initially to the index case and all the newly admitted infants remained not infected. This finding denotes that bundle of introduced measures were effective and adequate in spite of reopening the unit for new admission 4 days ahead of the control of viral outbreak. Our finding supports the proposal of Ben Shimol et al [11] that closing the neonatal units during parainfluenza-virus outbreak most probably is unnecessary.

We strongly think that nursing infectious infants inside closed incubators during parainfluenza viral outbreaks will have a positive role that adds up to the currently used infectious control measures to contain the virus spread in this population and shorten the outbreak duration.

Finally, our result confirms that infants with lower gestational age and or lower birth weight, infants with longer stay in the NCU, and infants with history of chronic lung disease, who were exposed to systemic steroid therapy and had longer period of mechanical ventilation have higher risk of PIV-3 infection during the outbreak.

\section{Conclusion}

Cohorting infected and exposed infants and placing them inside closed incubators could contain respiratory outbreaks in neonatal nurseries. Implementing infection control measures is essential to control viral respiratory outbreaks without significant morbidities or mortalities. Closure of the neonatal units during such outbreak may not be necessary.

\section{Acknowledgements}

The authors would like to thank Ms. Samia Mohamed Alsomali for her contribution in the manuscript preparation.

\section{Conflict of Interest Statement}

The authors disclose that we don't have any financial or personal relationships with other people or organizations that could inappropriately influence (bias) our work and we don't have any competing interest to declare.

\section{References}

[1] Parija S, Marrie T. Parainfluenza Virus. E Medicine. October 3, 2012. Accessed on December 25, 2013. Available at: http://www.emedicine.com/med/TOP_IC1733.htm.

[2] Human Parainfluenza Viruses. CDC web site. November 5, 2012.Accessed on December 25, 2013. Available at: http://www.cdc.gov/ncidod/dvrd/revb/respiratory/hpivfeat.htm.

[3] Henrickson KJ. Parainfluenza viruses. Clin Microbiol Rev 2003; 16: 242-64.

[4] Águeda S, Leitão A, Rocha G, Guimarães H. Viral Infections in a Neonatal Intensive Care Unit. Pediat Therapeut2013; 3: 154-57.

[5] Glezen WP, Frank AL, Taber LH, Kasel JA. Parainfluenza virus type 3: seasonality and risk of infection and reinfection in young children. J Infect Dis 1984; 150:851-57.

[6] Simmonds A, Munoz J, Montecalvo M, Clones B, Lagamma EF. Outbreak of parainfluenza virus type 3 in a neonatal intensive care unit. Am J Perinatol 2009; 26:361-64.

[7] Moisiuk SE, Robson D, Klass L,Kliewer G,Wasyliuk W,Davi M, Plourde P. Outbreak of parainfluenza virus type 3 in an intermediate care neonatal nursery. Pediatr Infect Dis J 1998; 17: 49-53.

[8] Gelber SE, Ratner AJ. Hospital-acquired Viral Pathogens in the Neonatal Intensive Care Unit. Sem in Perinatol, 2002; 26: 346-56. 
[9] Singh-Naz N, Willy M Riggs N. Outbreak of Parainfluenza virus type 3 in neonatal nursery. Pediatr Infec Dis J 1990; 9: 31-33.

[10] Teo WY, Rajadurai VS, Sriram B. Morbidity of Parainfluenza 3 Outbreak in preterm infants in a neonatal unit. Ann Acad Med Singapore 2010; 39:837-42.

[11] Ben-Shimol S, Landau D, Zilber S, Greenberg D. Parainfluenza virus type 3 outbreak in a neonatal nursery. Clin Pediatr 2013; 52: 866-70.

[12] Meissner HC, Murray SA, Keirnan MA, Snydman DR, McIntosh K. A. Simultaneous outbreak of respiratory syncytial virus and parainfluenza virus type 3 in a newborn nursery. J Pediatr 1984; 104: $680-84$
[13] Ansari SA, Springthorpe VS, Satter SA, Rivard S, Rahman M. Potential role of hands in the spread of respiratory viral infection. Studies with human parainfluenza virus type 3 and rhinovirus. $J$ clin Microbial 1991; 29: 2115-19.

[14] Brady MT, Evans J, Cuartas J. Survival and disinfection of parainfluenza viruses on environmental surfaces. Am J Infect Control 1990; 18:18-23.

[15] Parkinson AJ, Muchmore HG, Scott EN, Scott LV. Survival of human parainfluenza viruses in the South Polar environment. Appl Environ Microbiol 1983; 46: 901-5.

[16] De A, Silva C, Dias L, Baltieri SR, Rodrigues TT, Takagi NB, Richtmann R. Respiratory syncytial virus outbreak in neonatal intensive care unit: Impact of infection control measures plus palvizumab use. Antimicrob.Resist.infect.control. 2012; 1: 16-19. 